\title{
Comparison of genetic-algorithm and emissivity-ratio analyses of image data from OMEGA implosion cores
}

Cite as: Rev. Sci. Instrum. 79, 10E921 (2008); https://doi.org/10.1063/1.2966370

Submitted: 26 May 2008. Accepted: 09 July 2008. Published Online: 31 October 2008

T. Nagayama, R. C. Mancini, R. Florido, R. Tommasini, J. A. Koch, J. A. Delettrez, S. P. Regan, V. A. Smalyuk, L. A. Welser-Sherrill, and I. E. Golovkin

\section{ARTICLES YOU MAY BE INTERESTED IN}

Investigation of a polychromatic tomography method for the extraction of the threedimensional spatial structure of implosion core plasmas Physics of Plasmas 19, 082705 (2012); https://doi.org/10.1063/1.4743017

Spatial structure analysis of direct-drive implosion cores at OMEGA using $\mathrm{x}$-ray narrow-band core images

Review of Scientific Instruments 77, $10 E 320$ (2006); https://doi.org/10.1063/1.2229196

Multiobjective method for fitting pinhole image intensity profiles of implosion cores driven by a Pareto genetic algorithm

Review of Scientific Instruments 77, $10 F 525$ (2006); https://doi.org/10.1063/1.2338314

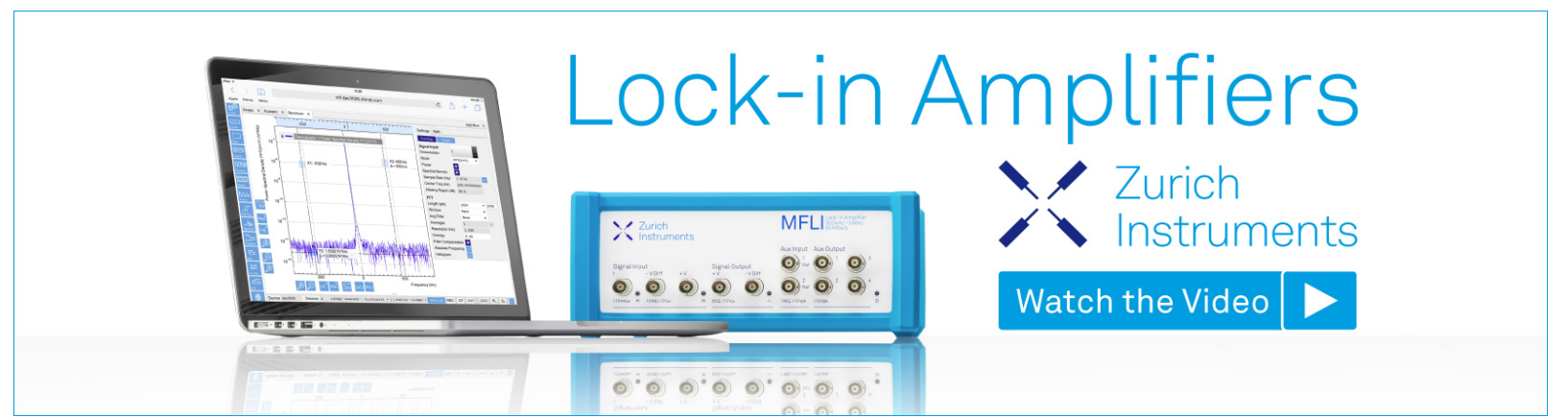




\title{
Comparison of genetic-algorithm and emissivity-ratio analyses of image data from OMEGA implosion cores ${ }^{\text {a) }}$
}

\author{
T. Nagayama, ${ }^{1}$ R. C. Mancini, ${ }^{1}$ R. Florido, ${ }^{1, b)}$ R. Tommasini, ${ }^{2}$ J. A. Koch, ${ }^{2}$ J. A. Delettrez, ${ }^{3}$ \\ S. P. Regan, ${ }^{3}$ V. A. Smalyuk, ${ }^{3}$ L. A. Welser-Sherrill, ${ }^{4}$ and I. E. Golovkin ${ }^{5}$ \\ ${ }^{1}$ Department of Physics, University of Nevada, Reno, Nevada 89557, USA \\ ${ }^{2}$ Lawrence Livermore National Laboratory, Livermore, California 94550, USA \\ ${ }^{3}$ Laboratory for Laser Energetics, University of Rochester, New York 14623, USA \\ ${ }^{4}$ Los Alamos National Laboratory, Los Alamos, New Mexico 87545, USA \\ ${ }^{5}$ Prism Computational Sciences, Madison, Wisconsin 53711, USA
}

(Presented 13 May 2008; received 26 May 2008; accepted 9 July 2008; published online 31 October 2008)

\begin{abstract}
Detailed analysis of x-ray narrow-band images from argon-doped deuterium-filled inertial confinement fusion implosion experiments yields information about the temperature spatial structure in the core at the collapse of the implosion. We discuss the analysis of direct-drive implosion experiments at OMEGA, in which multiple narrow-band images were recorded with a multimonochromatic $\mathrm{x}$-ray imaging instrument. The temperature spatial structure is investigated by using the sensitivity of the $\mathrm{Ly} \beta / \mathrm{He} \beta$ line emissivity ratio to the temperature. Three analysis methods that consider the argon $\mathrm{He} \beta$ and $\operatorname{Ly} \beta$ image data are discussed and the results compared. The methods are based on a ratio of image intensities, ratio of Abel-inverted emissivities, and a search and reconstruction technique driven by a Pareto genetic algorithm. (C) 2008 American Institute of Physics. [DOI: 10.1063/1.2966370]
\end{abstract}

\section{INTRODUCTION}

Determining the spatial structure of implosion core temperature conditions is of current interest in inertial confinement fusion experiments. ${ }^{1-5}$ We report here on the extraction of the temperature spatial structure from x-ray narrow-band gated images of the implosion core recorded in direct-drive experiments performed at the Laboratory for Laser Energetics' OMEGA laser facility. The temperature spatial profiles are determined spectroscopically using three methods that rely on the sensitivity of the $\mathrm{Ly} \beta / \mathrm{He} \beta$ line emissivity ratio with respect to the temperature. These methods treat space integration and radiation transport effects with different levels of approximation.

\section{EXPERIMENTAL DETAILS AND DATA}

The direct-drive experiments discussed here consisted of imploding spherical plastic microballoon targets irradiated with the OMEGA laser system. The microballoons had an initial radius of $400 \mu \mathrm{m}$, a plastic wall thickness of $27 \mu \mathrm{m}$, and an outer aluminum layer of $0.1 \mu \mathrm{m}$. They were filled with $20 \mathrm{~atm}$ of deuterium and a tracer amount of argon $(0.072 \mathrm{~atm})$ for spectroscopic purposes, and driven by 18.5 $\mathrm{kJ}$ of UV laser energy in a low-adiabat $\alpha 2$ pulse shape. This pulse shape begins with low laser power to drive a slow

\footnotetext{
${ }^{\text {a) }}$ Contributed paper, published as part of the Proceedings of the 17th Topical Conference on High-Temperature Plasma Diagnostics, Albuquerque, New Mexico, May 2008.

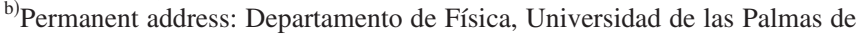
Gran Canaria, Las Palmas, Spain.
}

implosion which is then followed by high laser power in an effort to mitigate early core preheating and achieve higher compression.

In order to extract information on the temperature spatial structure of the implosion cores, good quality images of the argon line emission are necessary. To this end, the directdrive multimonochromatic x-ray imager (DDMMI) instrument was used to record argon $K$-shell line emission images in the photon energy range of interest, namely, the Ly $\beta$ $(1 s-3 p, h \nu=3935 \mathrm{eV})$ and $\mathrm{He} \beta\left(1 s^{2}-1 s 3 p, h \nu=3684 \mathrm{eV}\right)$ line transitions of $H$ - and He-like Ar ions, respectively. ${ }^{6}$ The DDMMI consists of a pinhole array placed as close as possible to the core in order to maximize the signal, and a multilayer mirror Bragg reflector to provide spectral dispersion. Time-resolved image data were recorded by time-gated framing cameras on film. The data presented here represent observations along one line of sight. However, images were also recorded along the two additional lines of sight from quasiorthogonal directions and will be reported elsewhere. In addition, two streaked $\mathrm{x}$-ray spectrometers were fielded to record time-resolved space-integrated $\mathrm{x}$-ray line emission from the argon tracer. A time-correlation technique based on the time history of the emission of $\operatorname{Ly} \alpha(1 s-2 p, h \nu$ $=3322 \mathrm{eV}$ ), $\mathrm{Ly} \beta$, and $\mathrm{He} \beta$ argon line transitions was used to establish a correspondence between the framing cameras and the continuous time axis of the streak cameras. Namely, the time-correlation function $h$ defined as

$$
h\left(t_{0}\right)=\int f_{\mathrm{SC}}(t) g_{\mathrm{FC}}\left(t-t_{0}\right) d t,
$$

where $f_{\mathrm{SC}}$ and $g_{\mathrm{FC}}$ refer to streak-camera and framingcamera recorded time histories, respectively, was computed 

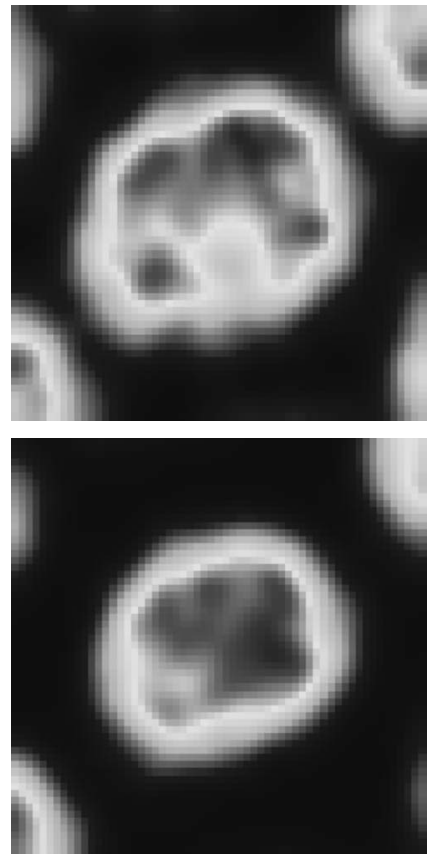

FIG. 1. Time-gated narrow-band core images, integrated over $\Delta t=50 \mathrm{ps,}$ based on the $\mathrm{He} \beta$ (top) and $\mathrm{Ly} \beta$ (bottom) argon line transitions.

and the time $t_{0}$ for which $h$ is maximum was used to correlate the time axis of $f_{\mathrm{SC}}$ and $g_{\mathrm{FC}}$. This was important in order to locate in time the gated core images recorded with DDMMI.

Raw data recorded by the DDMMI instrument were first converted from film density to intensity using the film calibration, and then corrected for the photon energy dependent corrections relating to beryllium filter transmission, reflectivity of the multilayer mirror, and the spectral response of the multichannel plates. The DDMMI instrument records many core images for each time frame. Implosion core images recorded by DDMMI associated with individual pinholes correspond to a slightly shifted photon energy range. Therefore, in order to compile a narrow-band spatial image based only on $\mathrm{Ly} \beta$ or $\mathrm{He} \beta$ line transition photons, it is necessary to extract, align, and combine several portions of DDMMI recorded images; details of this procedure are described in Ref. 6 . In this case, portions from about six images were carefully aligned and added to compile $\operatorname{Ly} \beta, \operatorname{He} \beta$, and nearby continuum composite images. The continuum images can then be subtracted from the $\operatorname{Ly} \beta$ and $\operatorname{He} \beta$ images in order to produce a good approximation to an image formed only by line transition photons.

As an illustration of the data recorded by DDMMI, a set of narrow-band images based on the $\operatorname{Ly} \beta$ and $\operatorname{He} \beta$ line transitions is shown in Fig. 1. Their narrow-band widths are approximately $60 \mathrm{eV}$ each, and they are integrated for a small time interval $\Delta t \approx 50$ ps close to the peak of argon $\mathrm{x}$-ray line emission at the collapse of the implosion. We note that the $\mathrm{He} \beta$ line core image displays an annular intensity distribution, and both $\operatorname{Ly} \beta$ and $\operatorname{He} \beta$ line images show visible asymmetries.

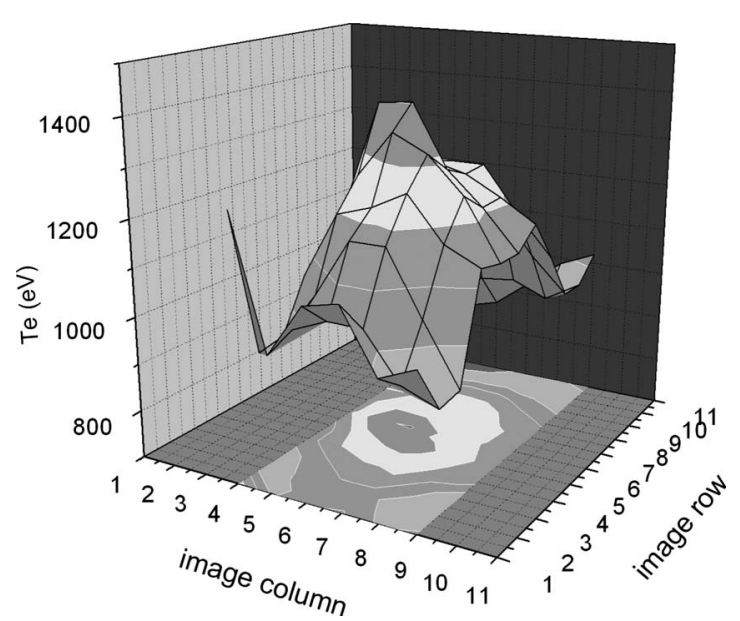

FIG. 2. Electron temperature $T_{e}$ spatial distribution obtained from the ratio of $\mathrm{Ly} \beta / \mathrm{He} \beta$ image intensities.

\section{SPECTROSCOPIC DETERMINATION OF ELECTRON TEMPERATURE SPATIAL STRUCTURE}

The emissivity ratio of $\mathrm{Ly} \beta / \mathrm{He} \beta$ is strongly dependent on electron temperature $T_{e}$ but only weakly dependent on electron density $N_{e}{ }^{5,7}$ Therefore, it can be used as a temperature diagnostic. Three methods were implemented to extract information about the spatial structure of the electron temperature $T_{e}$. The differences between these methods can be interpreted in terms of the way in which they deal with spatial integration and radiation transport effects. Emergent intensity distributions and emissivities and opacities required by these analysis methods were computed with a collisionalradiative atomic kinetics model that includes detailed Starkbroadened line profiles and spectroscopic quality radiation transport. ${ }^{8}$ The first method considers the ratio of $\mathrm{Ly} \beta / \mathrm{He} \beta$ image intensities. If we neglect the radiation transport effect, each intensity point on the image plane can be interpreted as the line integral of the emissivity along a given chord in the plasma source, i.e., the implosion core. Thus, the ratio of $\mathrm{Ly} \beta / \mathrm{He} \beta$ intensities on the image plane corresponds to the ratio of average emissivities along chords in the core, and this ratio can be converted into an average or effective electron temperature $T_{e}$ integrated along the chord. A constant electron density $N_{e}=1 \times 10^{24} \mathrm{~cm}^{-3}$ was assumed. The result of this analysis for the image data of Fig. 1 is displayed in Fig. 2. The $x$ and $y$ coordinates in the surface plot correspond to the coordinates on the image plane. The electron temperature $T_{e}$ varies in the range from $900 \mathrm{eV}$ near the core image edge to $1400 \mathrm{eV}$ in a region off image center. We note that no symmetry assumptions or geometry inversions are required to perform this analysis.

The second method splits the core into a collection of core slices and uses an Abel inversion procedure for each core slice to go from intensity distribution on the image plane to emissivity profile in the plasma (core) source. ${ }^{9}$ The Abel inversion unfolds the line integration corresponding to chords in each core slice to yield local emissivity in the core slice but it still assumes negligible radiation transport effects. Also, the application of the Abel inversion procedure requires local axial symmetry in each core slice. To satisfy this 


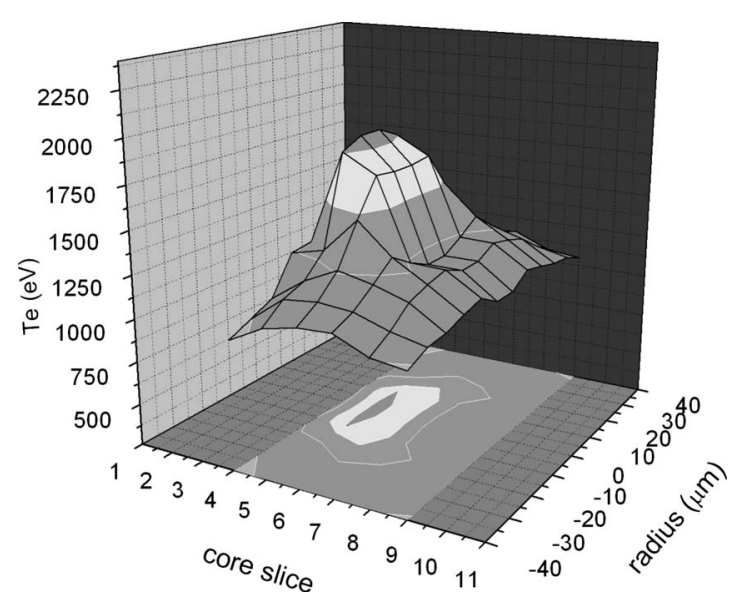

FIG. 3. Electron temperature $T_{e}$ spatial distribution obtained from the ratio of $\mathrm{Ly} \beta / \mathrm{He} \beta$ Abel-inverted emissivities.

condition intensity profiles associated with a given core slice must be made symmetric about a suitable center point. This operation partially removes some of the asymmetries observed on the image thus yielding Abel-inverted emissivities as a function of radial coordinate $r$ in the core slice. Then, the ratio of Abel-inverted emissivities can be converted into a spatial profile of $T_{e}$ as a function of the radial coordinate in the core slice, i.e., $T_{e}(r)$. A constant electron density $N_{e}=1$ $\times 10^{24} \mathrm{~cm}^{-3}$ was assumed. The collection of $T_{e}(r)$ profiles from all core slices represents a quasi-three-dimensional map of the electron temperature spatial distribution. The result of this analysis for the image data in Fig. 1 is shown in Fig. 3. The $x$ and $y$ coordinates in the surface plot now correspond to core slice label and radial coordinate in the core slice. While this analysis still neglects the radiation transport effect, the unfolding of the chord line integration via the Abel inversion results in an electron temperature that ranges from about $900 \mathrm{eV}$ near core edge to $1900 \mathrm{eV}$ inside the core but off the core center. This temperature distribution is axially symmetric in each core slice but it still has some of the asymmetry present in the image data. Finally, the third method is an attempt to unfold the chord line integration and to account for the radiation transport effect. As in the second method, the core is split into slices and each core slice is assumed to be axially symmetric. Then, a multiobjective Pareto genetic algorithm is used to drive a search in parameter space with the goal of finding the temperature and density profiles that yield the best simultaneous and selfconsistent fits (i.e., approximations) to three objectives: the $\mathrm{He} \beta$ image intensity distribution, the Ly $\beta$ image intensity distribution, and the space-integrated line spectrum. Alternatively, the $\mathrm{Ly} \beta / \mathrm{He} \beta$ image intensity ratio can be used as an objective as well. Furthermore, optimal fits are obtained by following up the genetic algorithm search with a "fine-tuner" step driven by a nonlinear least-squares minimization algorithm. ${ }^{10}$ The approximation of the image intensity distribution on the image plane is performed by transporting the line radiation, one photon energy at a time, inside the core slice via an integration of the radiation transport equation. The result of this analysis for the image data in Fig. 1. is shown in Fig. 4. Accounting for both space integration and

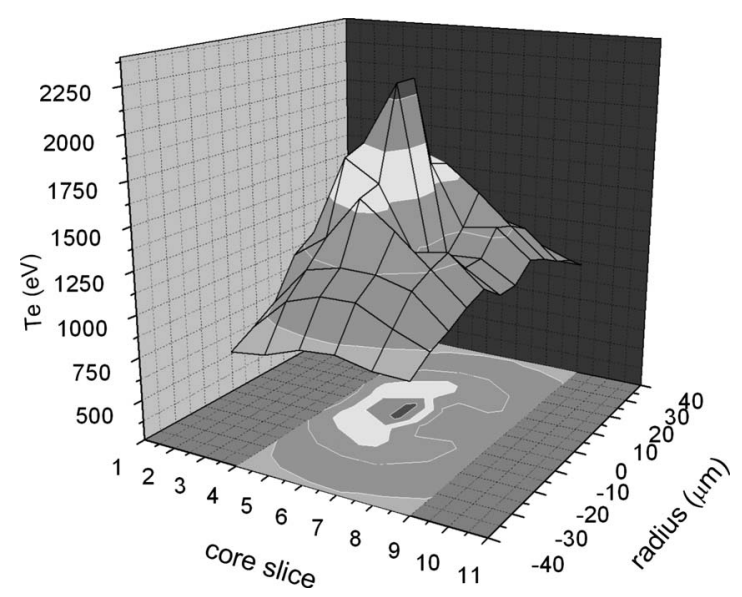

FIG. 4. Electron temperature $T_{e}$ spatial distribution obtained from a search and reconstruction method driven by a Pareto genetic algorithm.

radiation transport effects yields a temperature spatial distribution in the core that spans the range from $900 \mathrm{eV}$ near core edge to $2250 \mathrm{eV}$ inside the core, thus resulting in the temperature spatial structure with the steepest gradients.

\section{CONCLUSION}

We have implemented three spectroscopic methods to determine time-resolved temperature spatial structure in direct-drive inertial confinement fusion implosion cores at OMEGA. The targets consisted of plastic shells filled with deuterium gas and a tracer amount of argon for diagnostic purposes, and driven with a low-adiabat $\alpha 2$ pulse shape. The analysis methods rely on the temperature sensitivity of the $\mathrm{Ly} \beta / \mathrm{He} \beta$ line emissivity ratio and on gated image data recorded with the DDMMI instrument. The methods treat space integration and radiation transport effects with different levels of approximation. On the one hand, neglect of these effects leads to a temperature spatial structure characteristic of the shallowest spatial gradients. On the other hand, taking both effects into account yields a spatial structure with the steepest spatial gradients.

\section{ACKNOWLEDGMENTS}

This work was supported by the DOE-NLUF under Grant No. DE-FG52-07NA28062, and LLNL.

${ }^{1}$ I. E. Golovkin, R. C. Mancini, S. Louis, Y. Ochi, K. Fujita, H. Nishimura, H. Shiraga, N. Miyanaga, H. Azechi, R. Butzbach, I. Uschmann, E. Forster, J. Delettrez, J. A. Koch, R. W. Lee, and L. Klein, Phys. Rev. Lett. 88, 045002 (2002).

${ }^{2}$ S. P. Regan, J. A. Delettrez, R. Epstein, P. A. Jaanimagi, B. Yaakobi, V. A. Smalyuk, F. J. Marshall, D. D. Meyerhofer, W. Seka, D. A. Haynes, I. E. Golovkin, and C. F. Hooper, Phys. Plasmas 9, 1357 (2002).

${ }^{3}$ A. A. Hauer, N. D. Delamater, and Z. M. Koenig, Laser Part. Beams 9, 3 (1991).

${ }^{4}$ B. A. Hammel, C. J. Keane, D. R. Kania, J. D. Kilkenny, R. W. Lee, R. Pasha, R. E. Turner, and N. D. Delamater, Rev. Sci. Instrum. 63, 5017 (1992).

${ }^{5}$ C. J. Keane, B. A. Hammel, D. R. Kania, J. D. Kilkenny, R. W. Lee, A. L. Osterheld, L. J. Suter, R. C. Mancini, C. F. Hooper, and N. D. Delamater, Phys. Fluids B 5, 3328 (1993).

${ }^{6}$ N. Izumi, T. W. Barbee, J. A. Koch, R. C. Mancini, and L. A. Welser, Rev. Sci. Instrum. 77, 083504 (2006).

${ }^{7}$ L. A. Welser, R. C. Mancini, J. A. Koch, N. Izumi, S. J. Louis, I. E. 
Golovkin, T. W. Barbee, S. W. Haan, J. A. Delettrez, F. J. Marshall, S. P. Regan, V. A. Smalyuk, D. A. Haynes, and R. W. Lee, J. Quant. Spectrosc. Radiat. Transf. 99, 649 (2006).

${ }^{8}$ I. E. Golovkin and R. C. Mancini, J. Quant. Spectrosc. Radiat. Transf. 65, 273 (2000).
${ }^{9}$ K. Bockasten, J. Opt. Soc. Am. 51, 943 (1961).

${ }^{10}$ T. Nagayama, R. C. Mancini, L. A. Welser, S. Louis, I. E. Golovkin, R. Tommasini, J. A. Koch, N. Izumi, J. Delettrez, F. J. Mrshall, S. P. Regan, V. Smalyuk, D. Haynes, and G. Kyrala, Rev. Sci. Instrum. 77, 10F525 (2006). 